\title{
Corrosion Behavior of Parylene-Metal-Parylene Thin Films in Saline
}

\author{
W. Li ${ }^{\mathrm{a}}$, D. C. Rodger ${ }^{\mathrm{a}, \mathrm{b}}$, P. R. Menon ${ }^{\mathrm{a}}$, and Y. C. Tai ${ }^{\mathrm{a}}$ \\ ${ }^{a}$ Electrical Engineering, California Institute of Technology \\ Pasadena, CA 91125, USA \\ ${ }^{\mathrm{b}}$ Keck School of Medicine, University of Southern California \\ Los Angeles, CA 90033, USA
}

\begin{abstract}
In this paper, we study the corrosion behavior of parylene-metalparylene thin films using accelerated-lifetime soak tests. The samples under test are thin film resistors with a $200 \mathrm{~nm}$ layer of $\mathrm{Au}$ sandwiched by parylene- $\mathrm{C}$ on both sides, fabricated with parylenemetal skin technology. The samples are tested in hot saline both passively and actively, and different failure modes are observed using optical and electron-beam metrologies. Bubbles and delamination are first seen in the samples after 2 days of soaking under passive conditions, and followed by metal corrosion. While under active conditions, either bubbles or parylene breakdowns are observed depending on the thickness of parylene packaging. These results contribute to a better understanding of the failure mechanisms of parylene packaging in body fluids.
\end{abstract}

\section{Introduction}

In recent years, micro-electro-mechanical systems (MEMS) technology has been widely used in biomedical microsystem development for neural prosthetic applications, particularly retinal implantation. These devices, when implanted into human bodies, will be exposed to the internal biochemical environment of humans and attacked by various body fluids (1). Therefore, hermetic and biostable packaging is needed in order to protect the systems from the corrosive biochemical environment. Furthermore, the packaging has to be optically transparent and flexible to facilitate surgical manipulation and to prevent any undesired damage to the area of implantation. To meet these challenges, parylene, more specifically, parylene $\mathrm{C}$ has been proposed as a structural and packaging material for such applications because of its favorable mechanical and chemical properties (2), including its pinhole-free conformality, low water permeability, chronic implantability, high flexibility and mechanical strength (Young's modulus $\sim 4 \mathrm{GPa}$ ). In fact, parylene-metal skin technology (3) has been well established in our lab and used to microfabricate MEMS devices such as microelectrode arrays and micro RF coils (4) (5) for biomedical applications. Although researches have been done to evaluate the longterm biostability of parylene packaging in the past years (6) (7), their failure mechanisms have not been well studied and fully understood. In this paper, we use acceleratedlifetime soak tests to study the corrosion behavior of parylene-metal-parylene thin film in hot saline, employing parylene packaged "Z-shaped" thin film resistors. The failure mechanisms of samples under different test conditions are also analyzed and discussed in the paper. 


\section{Experimental Method}

\section{$\underline{\text { Device Fabrication }}$}

Devices used in experiments consist of a layer of metal sandwiched by two layers of paryelen C, as depicted in Figure 1(a). Their detailed fabrication process is described in Figure 1(b). Briefly, photoresist was spun on a silicon substrate as a sacrificial layer. Then a layer of parylene with various thicknesses was deposited on top of the photoresist. After parylene deposition, a $200 \mathrm{~nm}$ layer of gold was e-beam evaporated and patterned to form the "Z-shaped" thin film resistors using a wet metal etching process. Another layer of parylene was then deposited and etched in a reactive-ion etching (RIE) system to properly open the contact vias, as well as to define the device shape with a photoresist mask. Finally, the completed devices were released from the substrate by dissolving the sacrificial photoresist in acetone. In order to investigate the thickness effect on parylene packaging, two sets of samples are prepared with different thicknesses of parylene: samples from set \#1 had $\sim 4.7 \mu \mathrm{m}$ parylene on each side, and samples in set \#2 had $\sim 9.2$ $\mu \mathrm{m}$ parylene on each side.

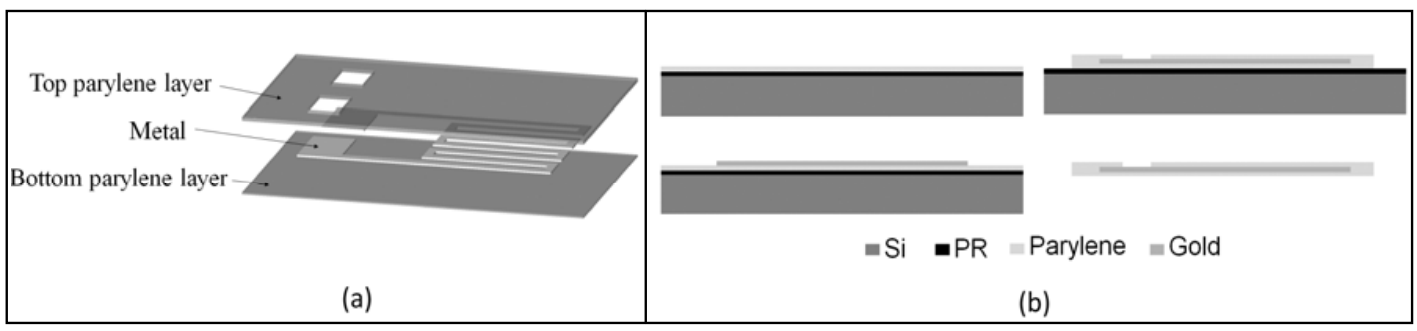

Figure 1. (a) Schematic of a parylene-metal-parylene thin film structure. (b) Detailed fabrication process using surface MEMS technology.

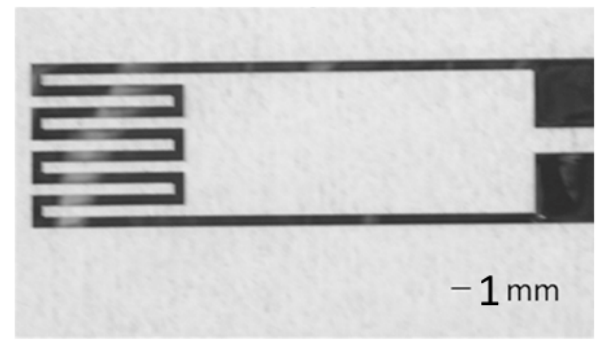

(a)

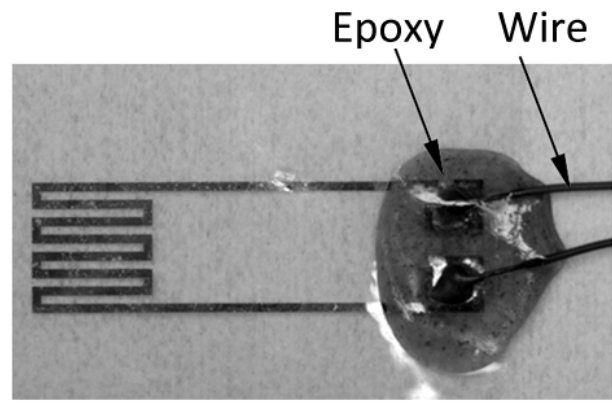

(b)

Figure 2. (a) A fabricated device with $10 \mu \mathrm{m}$ parylene $\mathrm{C}$ on each side. (b) A device with bonding wires.

Figure 2(a) shows a fabricated device with dimensions of approximately $1 \mathrm{~cm}$ by $3 \mathrm{~cm}$. In order to connect the devices to peripheral equipments for resistance testing, wires were glued on the contact openings using conductive silver epoxy and sealed by insulating epoxy to avoid undesired corrosion on the contact vias (Figure 2(b)). 


\section{$\underline{\text { Accelerated-Lifetime Test }}$}

Samples were tested under both active and passive soak conditions, i.e. with and without electrical drive signals applied. All tests were performed in saline $(0.9 \% \mathrm{NaCl}$ solution), which is a close imitator of body fluids. The solution was replaced once a week to maintain a constant concentration of $\mathrm{NaCl}$. During the passive soak tests, devices were immersed in saline and heated at $90{ }^{\circ} \mathrm{C}$ in a convection oven. Their DC resistances were recorded with an HP 34401A multimeter at 10-minute intervals. To monitor their failure mechanisms, the samples were removed from the oven every day and examined under a microscope. Only samples from set \#1 were tested under passive conditions.

For active soak tests, a simplified electrochemical test setup was built, as illustrated in Figure 3. The soak cell consisted of two electrodes, in which a platinum wire served as a reference electrode and the device was used as a working electrode. A square wave with a magnitude of 6 Volts and a frequency of $50 \mathrm{~Hz}$ was applied on the working electrode using a function generator. Then the entire test cell was placed in the oven and heated up to $90{ }^{\circ} \mathrm{C}$. The DC resistances of the devices were measured every 10 minutes using an HP 4145B controlled by a LabView program. Samples from both sets were tested under active conditions. These tests continued until failures were observed, and then the failed samples were inspected using both optical and electron-beam metrologies in order to determine their failure modes.

\section{Function generator}

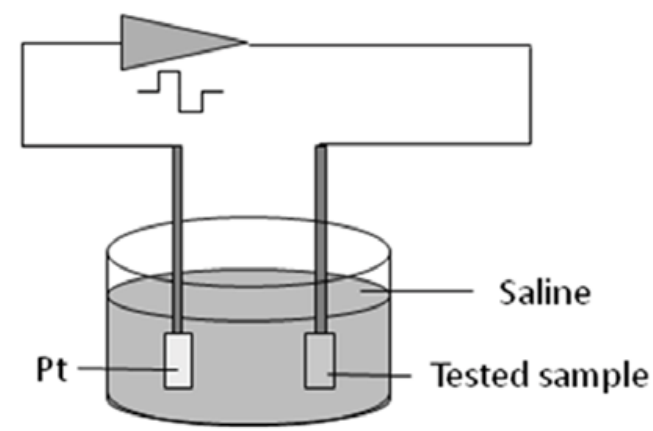

Figure 3. Simplified electrochemical setup of active soak tests.

\section{Results and Discussions}

Bubbles and delamination were first observed in all samples after 1 or 2 days of soaking under passive conditions, as shown in Figure 4(a). A typical curve of measured resistances during the soaking period is given in Figure 4 (b), which shows that the initial resistance was around $60 \Omega$, and increased gradually after 80 hours of soaking. This phenomenon indicated that moisture diffused through and attacked the interface between metal and parylene at the beginning. Subsequently, the metal got corroded in saline which caused the resistance to increase. Although the mechanism has not been fully understood, one possible diffusion path is believed to be the inhomogeneous interface between the two parylene layers. When the samples were removed from vacuum chamber after the first parylene deposition, the parylene surface was exposed and 
contaminated, which inhibited continuous polymerization on the strands of parylene molecules. This discrete parylene layer could not chemically react with additional parylene in the following deposition, and therefore resulted in an inhomogeneous interface. In addition, it is possible that unpyrolyzed molecules and particles were induced and stuck in parylene films during deposition, which cannot be removed easily. These contaminants could create additional diffusion paths for moisture during soaking.

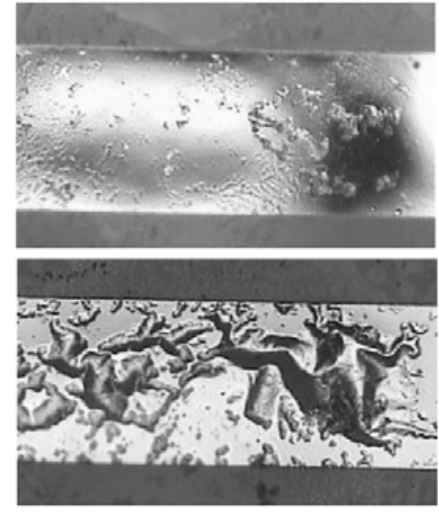

(a)

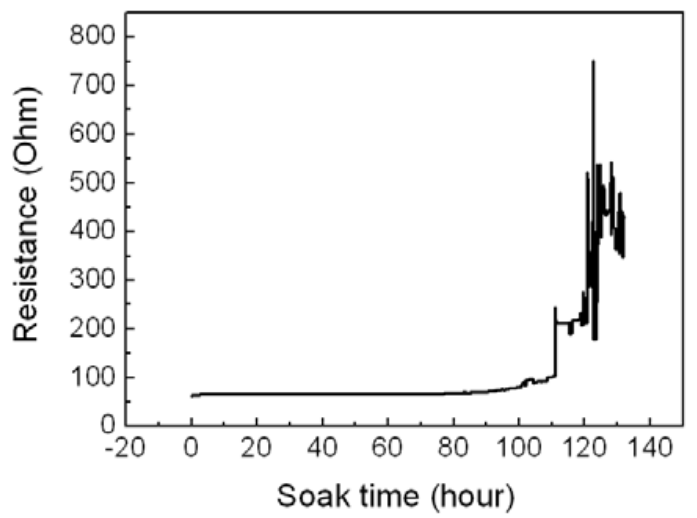

(b)

Figure 4. (a) Optical images of bubbles and delamination from a failed sample. (b) Aging curve of resistance for a sample under passive soak conditions.

In the active soak tests, different failure mechanisms were observed in samples with different parylene thicknesses. For the samples with $4.7 \mu \mathrm{m}$ on each side, breakdown holes were found to localize in certain areas when the failed samples were inspected in a scanning electron microscope (SEM). Energy dispersive X-ray spectroscopy (EDS) analysis was also performed in the breakdown areas, which showed that through holes were created in the parylene film, and the metal was directly exposed to saline (Figure 5). This failure was probably due to high electric field concentration in areas containing defects or particles. Then the parylene was melted by local heating, and electrochemical reactions were accelerated at those defect points, which resulted in open circuits in the metal resistors. This is verified by the resistance curve measured during soak tests, as
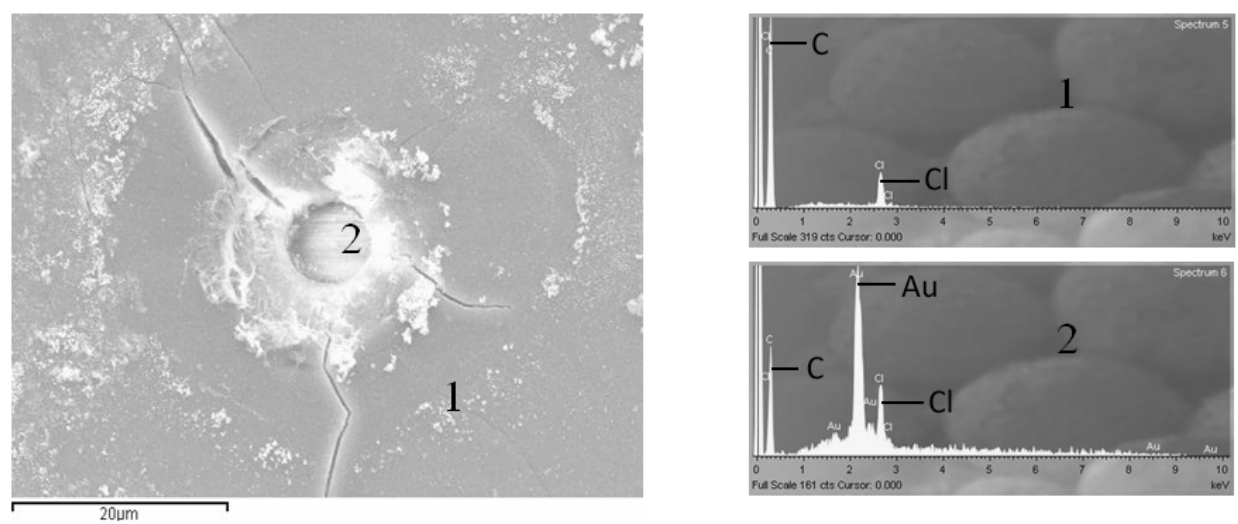

Figure 5. SEM/EDS images of parylene breakdown found in a sample with $4.7 \mu \mathrm{m}$ parylene on both sides. 
presented in Figure 6(a). After 70 hours of soaking, there was a dramatic rise in resistance from $25 \Omega$ to over $400 \mathrm{k} \Omega$, which is close to what one would expect from the saline solution. When the sample was removed from saline, the resistance was measured as an open circuit.

For samples with thicker parylene packaging, only bubbles were observed uniformly across the whole device, which indicated that thicker parylene could cover defects or particles, and therefore, moisture diffusion became the dominant failure mechanism instead of parylene breakdown. Figure 6(b) shows a representative curve from sample set $\# 2$, in which the resistance was stable during the first 55 days of soaking, and increased significantly after 65 days. Furthermore, the testing results indicated that thicker parylene film had longer lifetime under accelerated soak conditions because of its better moisture barrier properties. However, these short term tests are still preliminary. Longterm continuous soak tests on specially treated parylene-based skins are needed to assist further understanding of the corrosion behavior and reliability of parylene packaging.

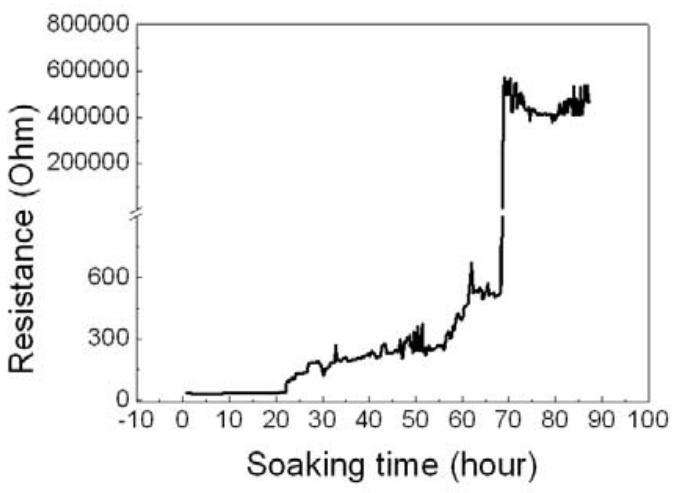

(a)

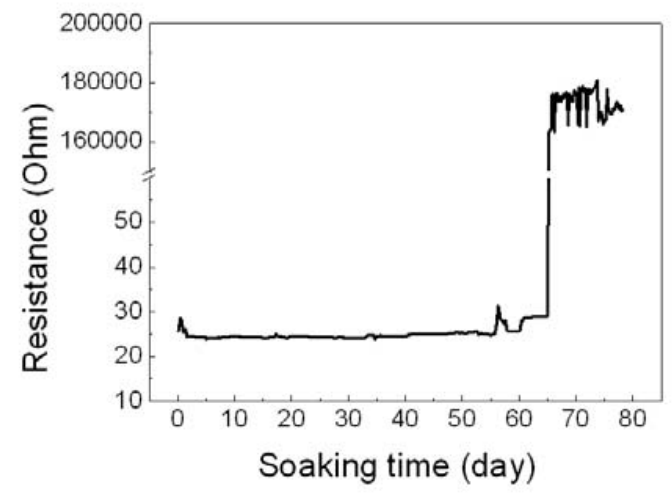

(b)

Figure 6. Typical aging curve of a sample: (a) with $4.7 \mu \mathrm{m}$ parylene C; (b) with $9.2 \mu \mathrm{m}$ parylene $\mathrm{C}$.

\section{Conclusions}

In this paper, corrosion behaviors of parylene-metal-parylene thin films were studied under accelerated-lifetime soak conditions, utilizing parylene packaged metal thin film resistors. Two sets of samples with different thicknesses of parylene packaging were tested in hot saline at $90{ }^{\circ} \mathrm{C}$ both passively and actively, and their failure mechanisms were analyzed and characterized. The preliminary test results show that moisture diffusion through parylene films or inhomogeneous interfaces was the main failure mechanism for parylene packaging under accelerated soak conditions. Parylene moisture barrier performance could be improved by increasing the thickness of parylene film. For parylene films with thicknesses less than $5 \mu \mathrm{m}$, parylene breakdowns were observed under high electric field due to contaminants or particles trapped inside the films. Although our test results are preliminary, they lead to a new path of evaluating the packaging characteristics of parylene for biomedical applications. 


\section{Acknowledgments}

This work was supported in part by the Engineering Research Center Program of the National Science Foundation under Award Number EEC-0310723 and by a fellowship from the Whitaker Foundation (D.R.). The authors would also like to thank Mr. Trevor Roper and other members in the Caltech Micromachining Laboratory for their assistance with fabrication.

\section{References}

1. U. Kamachi Mudali, T. M. Sridhar, and Baldev Raj, $S^{-}$adhan ${ }^{-} a, 28,601$ (2003).

2. L. Wolgemuth, Medical Device \& Diagnostic Industry, 22, 42 (2000).

3. Y. C. Tai, F. K. Jiang, Y. Xu, M. Liger, S. Ho, and C. M. Ho, Proc. Pacific Rim MEMS Workshop, Xiamen, China, (2002).

4. D. C. Rodger, W. L, H. Ameri, A. Ray, J. D. Weiland, M. S. Humayun, and Y. C. Tai, Proc. IEEE-NEMS 2006, Zhuhai, China (2006).

5. W. Li, D. C. Rodger, E. Meng, J. D. Weiland, M. S. Humayun, and Y. C. Tai, Proc. IEEE-EMBS Special Topic Conference on Microtechnologies in Medicine and Biology, Okinawa, Japan, 105 (2006).

6. T. Stieglitz, $10^{\text {th }}$ Annual Conference of the International FES Society, Montreal, Canada, (2005).

7. M. Kazemi, E. Basham, M. Sivaprakasam, G. Wang, D. C. Rodger, J. D. Weiland, Y. C. Tai, W. Liu and M. Humayun, Proc. IEEE-EMBS 2004, San Francisco, CA, 4093 (2004). 